\title{
Mannose-binding lectin deficiency is associated with early onset of polyarticular juvenile rheumatoid arthritis: a cohort study
}

\author{
Koert M Dolman ${ }^{1,2}$, Nannette Brouwer ${ }^{2}$, Florine NJ Frakking ${ }^{1}$, Berit Flat $\varnothing^{3}$, Paul P Tak ${ }^{4}$ \\ Taco W Kuijpers ${ }^{1,2}, \varnothing y s t e i n$ Førre $^{3}$ and Anna Smerdel-Ramoya ${ }^{3}$
}

\author{
1'Department of Pediatric Hematology, Immunology and Infectious diseases, Emma Children's Hospital, Academic Medical Center, University of \\ Amsterdam, Meibergdreef, Amsterdam, 1105 AZ, The Netherlands \\ 2Department of Blood Cell Research, Sanquin Research at CLB, and Landsteiner Laboratory, University of Amsterdam, Plesmanlaan, Amsterdam, \\ $1066 \mathrm{CX}$, The Netherlands \\ ${ }^{3}$ Department of Rheumatology, Rikshospitalet University Hospital, Sognsvannsveien, Oslo, NO-0027, Norway \\ ${ }^{4}$ Division of Clinical Immunology and Rheumatology, Academic Medical Center, University of Amsterdam, Meibergdreef, Amsterdam, 1105 AZ, The \\ Netherlands \\ Corresponding author: Florine NJ Frakking, f.n.frakking@amc.uva.nl
}

Received: 18 Dec 2007 Revisions requested: 6 Feb 2008 Revisions received: 29 Feb 2008 Accepted: 11 Mar 2008 Published: 11 Mar 2008

Arthritis Research \& Therapy 2008, 10:R32 (doi:10.1186/ar2386)

This article is online at: http://arthritis-research.com/content/10/2/R32

(C) 2008 Dolman et al.; licensee BioMed Central Ltd.

This is an open access article distributed under the terms of the Creative Commons Attribution License (http://creativecommons.org/licenses/by/2.0), which permits unrestricted use, distribution, and reproduction in any medium, provided the original work is properly cited.

\begin{abstract}
Background Mannose-binding lectin (MBL) is an innate immune protein. The aim of our study was to determine whether genetically determined MBL deficiency is associated with susceptibility to juvenile rheumatoid arthritis (JRA) and whether MBL2 genotypes are associated with JRA severity.

Methods In a retrospective cohort study of 218 patients with polyarthritis $(n=67)$ and oligoarthritis $(n=151)$, clinical and laboratory disease variables were obtained by clinical examination and chart reviews. Healthy Caucasian adults $(\mathrm{n}=$ 194) served as control individuals. MBL2 gene mutations were determined by Taqman analysis to identify genotypes with high, medium and low expression of MBL. Functional MBL plasma concentrations were measured using enzyme-linked immunosorbent assay. Associations between clinical and laboratory variables and $M B L 2$ genotypes were determined by Kruskal-Wallis and $\chi^{2}$ tests.
\end{abstract}

Results MBL2 genotype frequencies were similar in polyarthritis and oligoarthritis patients as compared with control individuals. MBL plasma concentrations were associated with the high, medium and low MBL genotype expression groups $(P$ $<0.01$ ). In polyarthritis patients, the presence of low-expressing (deficient) MBL2 genotypes was associated with early age at onset of disease $(P=0.03)$. In oligoarthritis patients, patients with low-expressing MBL2 genotypes were more often in remission (81\%) than patients in the medium (54\%) and high (56\%) genotype groups $(P=0.02)$. The remaining clinical and laboratory variables, such as arthritis severity index, presence of radiographic erosions and antinuclear antibody positivity, were not associated with $M B L 2$ genotypes.

Conclusion Genetically determined MBL deficiency does not increase susceptibility to JRA, but MBL deficiency is associated with a younger age at onset of juvenile polyarthritis. On the other hand, MBL-deficient children with juvenile oligoarthritis are more often in remission. Therefore, MBL appears to play a dual role in JRA.

\section{Introduction}

Juvenile rheumatoid arthritis (JRA), also known as juvenile idiopathic arthritis (JIA), is a rheumatic disease of childhood, and includes a heterogeneous group of patients with differing characteristics, clinical manifestations, serological parameters and genetic background. Although the aetiology of JRA remains unknown, it appears to be a combined action of environmental, hormonal and genetic factors [1-3]. It is generally believed that infections play an important role in the pathogenesis of JRA [4].

$\overline{\mathrm{ANA}}=$ antinuclear antibody; $\mathrm{CHAQ}=$ Childhood Health Assessment Questionnaire; $\mathrm{CRP}=\mathrm{C}$-reactive protein; $\mathrm{IQR}=$ interquartile range; $\mathrm{MBL}=$ mannose-binding lectin; JIA = juvenile idiopathic arthritis; JRA = juvenile rheumatoid arthritis; PGA = physician's global assessment; RA = rheumatoid arthritis; RF = rheumatoid factor; SNP = single nucleotide polymorphism. 
Mannose-binding lectin (MBL) is a serum protein, produced in the liver, that plays an important role in innate immunity and functions as an opsonin, recognizing sugar structures on a wide variety of micro-organisms [5]. Serum MBL can directly opsonize micro-organisms and enhance the uptake by phagocytic cells via activation of the lectin pathway of the complement system [6,7]. Genetically determined functional MBL serum levels vary within the population. Six single nucleotide polymorphisms (SNPs) in the MBL2 gene on chromosome 10 are known to influence MBL plasma levels. Reduced or deficient MBL plasma levels are seen in individuals with heterozygous or homozygous SNPs in codons 54 ( $B$ mutation), 52 ( $D$ mutation), or 57 ( $C$ mutation) of exon 1 of the $M B L 2$ gene $[5,8,9]$. The variant alleles occur with a combined phenotype frequency of about $25 \%$ to $30 \%$ in the Caucasian population $[10,11]$. The wild-type is called $A$, whereas the common designation for the variant alleles is $O$. In addition, MBL plasma concentrations fluctuate in the presence or absence of three SNPs (position -550: $H$ and $L$ alleles; position -221: $X$ and $Y$ alleles; and position $+4: P$ and $Q$ alleles) in the promoter region of the $M B L 2$ gene $[12,13]$. However, only the $X / Y$ variant has a pronounced influence; the $X$ allele is associated with decreased plasma MBL levels and the $Y$ variant with high plasma MBL levels. Subsequently, intermediately decreased MBL serum levels are seen in individuals with the genotypes $X A / X A$ and $Y A / O$, whereas very low or undetectable serum $\mathrm{MBL}$ levels are seen in individuals with genotypes $X A / O$ and $O / O$. Individuals with $Y A / Y A$ and $Y A / X A$ haplotypes have high or normal MBL levels. Therefore, patients can be classified into high $(Y A / Y A$ and $Y A / X A)$, medium $(X A / X A$ and $Y A / O)$ and low $(X A / O$ and $O / O) \mathrm{MBL}$ genotype expression groups $[10,14]$.

MBL deficiency has been associated with increased susceptibility to and severity of infections, especially in children $[15,16]$. In addition, it has been suggested that MBL modulates inflammation and autoimmune disease; for example, variant MBL alleles are risk factors for systemic lupus erythematosus $[17,18]$. It has also been suggested that MBL deficiency is associated with joint erosions and early disease onset of adult rheumatoid arthritis (RA) [19-23], although other investigators were unable to confirm such an association $[24,25]$. Moreover, it is believed that MBL plays an important role in innate immunity. Although unproven, it has been hypothesized that infection may trigger JRA in genetically susceptible patients [26]; this viewpoint suggests that MBL deficiency can predispose to JRA. In a recently reported study [27], there was no significant difference in genotypic frequencies of $M B L 2$ codon 54 SNPs between 93 patients with JIA and 48 healthy control individuals. Codon 57 SNPs were not found. The other $M B L 2$ SNPs were not investigated in this study. In addition, no association of MBL2 haplotypes was found between the subgroups of patients with JIA and control individuals.

The aim of the present study was to determine whether genetically determined MBL deficiency is associated with suscepti- bility to JRA and whether MBL2 genotypes are associated with severity of JRA, as assessed based on patient characteristics and disease variables.

\section{Materials and methods Patients and samples}

Eligible patients participated in a larger cohort study of Caucasian Norwegian children with JRA and visited the Department of Rheumatology of Rikshospitalet University Hospital (Oslo, Norway) for the first time between January 1980 and September $1985[28,29]$. JRA was defined as meeting the American College of Rheumatology criteria for JRA [30]. The 236 patients from whom blood was drawn were stratified according to JRA subgroup, because disease variables vary within these groups. Patients with systemic arthritis $(n=2)$ and juvenile spondylarthropathy (juvenile ankylosing spondylitis [ $=3]$, seronegative enthesopathy [ $n=4]$, juvenile psoriatic arthritis $[n=11]$, or inflammatory bowel disease associated arthritis [ $=1]$ ) were excluded because these subgroups consisted of too few individuals to permit reliable statistic analysis. Of the 218 remaining patients, 151 had oligoarthritis and 67 had polyarthritis. The patients were examined and interviewed after a median disease duration of 14.8 years (interquartile range [IQR] 13.5 to 16.2 years) and their medical records were reviewed for variables associated with the onset and course of disease.

Plasma samples were immediately frozen at $-80^{\circ} \mathrm{C}$. Genomic DNA was isolated from heparinized/EDTA blood according to standard procedures. The study is compliant with the Helsinki Declaration. It was approved by the Regional Ethics Committee for Medical Research and written informed consent was given by the parents. Routine laboratory investigations included C-reactive protein (CRP) level and erythrocyte sedimentation rate, and detection of IgM-rheumatoid factor (RF) and antinuclear antibodies (ANAs). In addition, MBL plasma concentrations and genotypes were determined in 194 healthy adult volunteers, who served as control individuals [10].

\section{Clinical data}

Demographic and clinical outcome variables were recorded from the charts at the follow-up visit. Onset of disease was defined as the date that arthritis was documented by a physician for the first time. The clinical examination included a physician's global assessment (PGA) of overall disease activity (ranging from 0 to 5 ) as well as assessment of numbers of actively involved (swollen or tender and mobility-restricted) and affected (swollen or mobility-restricted) joints, disease remission status (current remission, active disease after previous remission, or continuously active disease) and presence of uveitis. Furthermore, the number of cumulative affected joints and the arthritis severity index score were recorded. The Childhood Health Assessment Questionnaire (CHAQ) was used to measure physical disability at follow up [31]. It 
measures physical functioning in the following areas: dressing and grooming, arising, eating, walking, hygiene, reaching, gripping and activities. The mean $\mathrm{CHAQ}$ score ranges from 0 to 3 , where 0 represents no disability and values above 1.5 represent severe disability.

\section{Radiographic examinations}

Radiographs of the sacroiliac joints, hips, ankles and tarsi were obtained at follow up of all patients, and examined by two radiologists, who were blinded to patient information and had no access to earlier radiographic, clinical, or laboratory data. Radiographs of other affected joints were obtained when clinically indicated. The radiographic changes were classified as joint erosions (grades III to V) or no joint erosions (grades 0 to II).

\section{MBL assays}

MBL measurements were performed at Sanquin Research and the Landsteiner Laboratory (Academic Medical Center, Amsterdam, The Netherlands). MBL plasma levels were measured using an enzyme-linked immunosorbent assay, as previously described [14,32]. Briefly, mannose was coated to the solid phase, and after incubation with plasma, biotinylated mouse-anti-human MBL lgG $(10 \mu \mathrm{g} / \mathrm{ml}$; Tacx and coworkers [32], Amsterdam) was used as detection antibody [32].

Genotyping of the promoter polymorphisms and exon 1 SNPs was performed by allelic discrimination using a Taqman assay, using specific primers and minor groove binding probes for each SNP [14,33]. Genotyping was performed independently of the clinical data collection and MBL plasma level measurements. Patients were classified into three MBL2 genotype groups with high, medium and low expression of MBL. The influence of the $X I Y$ allele was also determined by studying six 'extended' genotype groups: $Y A / Y A, Y A / X A, X A / X A, Y A / O$, $X A / O$ and $O / O$.

\section{Statistical analysis}

Data are presented as median and IQR because clinical and laboratory variables were not normally distributed. Consequently, the nonparametric Kruskal-Wallis and Mann-Whitney $\mathrm{U}$ tests were used for comparison of these variables. Frequencies between groups were compared by the $\chi^{2}$ or Fisher's exact test, where appropriate. Multivariate binominal logistic regression was used to study the association between MBL2 genotype and remission status (active/remission) after adjustment for disease duration. The odds ratio and $95 \%$ confidence interval were calculated. $P<0.05$ was considered statistically significant. Patients were stratified according to remission status (active/remission) to explore further the association between CRP levels and MBL2 genotype in oligoarthritis patients. For statistical analysis SPSS 12.0.1 software was used (SPSS Inc., Chicago, IL, USA).

\section{Results Demographics}

The patient group consisted of 59 boys (27\%) and 159 girls (73\%), with a median age at diagnosis of 8.0 years (range 0.8 to 15.4 years; Table 1 ). The median (IQR) follow-up time was 14.8 (13.6 to 16.2) years. Table 1 shows that most patient characteristics differ between polyarthritis and oligoarthritis patients $(P<0.05)$. Therefore, the association between MBL2 genotype and disease was analyzed in the two JRA subsets separately (see below).

\section{MBL genotype and functional MBL levels in relationship to disease}

The median (range) $\mathrm{MBL}$ plasma concentration was 1.23 (0.01 to 7.59$) \mu \mathrm{g} / \mathrm{ml}$ in the $218 \mathrm{JRA}$ patients. Frequencies of the $B, C$ and $D$ exon 1 mutations in these JRA patients did not differ significantly from those in control individuals $(P=0.89$, $P=1.00$ and $P=0.37$, respectively; Table 2$)$. No deviation from Hardy-Weinberg equilibrium was observed in JRA patients or healthy control individuals (data not shown). Of the 218 JRA patients, $113(52 \%)$ were in the high genotype expression group, 71 (33\%) were in the medium genotype group and $34(16 \%)$ were in the low genotype expression group (Table 2). The frequency of MBL deficiency was similar in JRA patients and control individuals (odds ratio 1.1, 95\% confidence interval 0.9 to $1.4 ; P=0.37$ ). The distribution of the extended MBL2 haplotypes in the 218 JRA patients was as follows: 62 (28\%) YA/YA haplotype, 51 (23\%) $Y A / X A$ haplotype, 15 (7\%) XA/XA haplotype, 56 (26\%) YA/O haplotype, 25 (12\%) XA/O haplotype and 9 (4\%) O/O haplotype. These frequencies did not differ significantly from those in control individuals $(P=0.89)$ or between the two JRA subgroups ( $P$ $=0.69$ ). $\mathrm{MBL}$ plasma concentrations were highest in the $Y A /$ $Y A$ genotype group and almost absent in $X A / O$ and $O / O$ groups (Figure 1). In JRA patients with high, medium and low expressing haplotypes, the median (IQR) MBL plasma level was 1.86 (1.23 to 3.26$) \mu \mathrm{g} / \mathrm{ml}, 0.77$ (0.38 to 1.41$) \mu \mathrm{g} / \mathrm{ml}$ and $0.07(0.04$ to 0.15$) \mu \mathrm{g} / \mathrm{ml}$, respectively $(P<0.01$; Table 2$)$. The MBL plasma concentrations of the six extended genotype groups did not differ between polyarthritis and oligoarthritis patients $(P>0.46)$.

\section{MBL association with disease parameters}

\section{Polyarthritis group}

In the 67 patients with polyarthritis, patients in the low MBL2 genotype group were younger (4.4 years, IQR 3.6 to 7.0 years) at onset of disease than the patients in the medium (10.1 years, IQR 8.4 to 13.0 years) and high (9.5, IQR 5.6 to 13.0 years) genotype groups $(P=0.05$; Table 3$)$. This association was even stronger after exclusion of the $11 \mathrm{lgM}-\mathrm{RF}$ positive patients $(P=0.02$; data not shown). The same association was found in the ANA-negative $(P<0.01)$ but not in the ANA-positive patients ( $P=0.47$; data not shown). In the high genotype expression group, four patients (11\%) were IgM-RF positive, as compared with seven patients (30\%) in 
Table 1

Demographic, clinical, and laboratory characteristics of JRA patients, according to disease onset subtype

\begin{tabular}{|c|c|c|c|c|}
\hline \multirow[t]{2}{*}{ Characteristic } & \multirow[t]{2}{*}{ All JRA patients $(n=218)$} & \multicolumn{2}{|c|}{ JRA subgroups } & \multirow[t]{2}{*}{$P$} \\
\hline & & Polyarthritis $(n=67)$ & Oligoarthritis $(n=151)$ & \\
\hline \multicolumn{5}{|l|}{ Demographic variables } \\
\hline Males $(n[\%])$ & $59(27 \%)$ & $19(28 \%)$ & $40(27 \%)$ & 0.87 \\
\hline Age (years) at onset & $8.0(3.7$ to 11.6$)$ & $9.4(5.5$ to 12.9$)$ & 7.3 (3.1 to 11.5$)$ & $<0.01$ \\
\hline Disease duration (years) at follow up & $14.8(13.6$ to 16.2$)$ & $14.6(13.4$ to 16.3$)$ & $15.0(13.8$ to 16.2$)$ & 0.68 \\
\hline \multicolumn{5}{|l|}{ Clinical variables } \\
\hline Number of cumulative affected joints & 5 (2 to 15$)$ & 20 (11 to 34$)$ & $4(2$ to 6$)$ & $<0.01$ \\
\hline Arthritis severity index & $2(0$ to 11$)$ & 12 (2 to 37 ) & $2(0$ to 5$)$ & $<0.01$ \\
\hline Physician global assessment & 1 (1 to 2$)$ & 2 (1 to 3$)$ & 1 (1 to 2$)$ & $<0.01$ \\
\hline Childhood Health Assessment Questionnaire score & $0(0$ to 0.4$)$ & $0.1(0$ to 0.6$)$ & 0 (0 to 0.3$)$ & $<0.01$ \\
\hline Patients with uveitis ( $n$ [\%]) & $42(19 \%)$ & $10(15 \%)$ & $34(23 \%)$ & 0.27 \\
\hline \multicolumn{5}{|l|}{ Remission status at follow-up ( $n[\%]$ ) } \\
\hline Current remission & $122(56 \%)$ & $32(48 \%)$ & $90(60 \%)$ & $<0.01$ \\
\hline Active but previous remission & $55(25 \%)$ & $14(21 \%)$ & $41(27 \%)$ & \\
\hline Continuously active & $41(19 \%)$ & $21(31 \%)$ & $20(13 \%)$ & \\
\hline Radiographic erosions grade III to IV ( $n$ [\%]) & $51(23 \%)$ & $30(45 \%)$ & $21(14 \%)$ & $<0.01$ \\
\hline \multicolumn{5}{|l|}{ Laboratory variables } \\
\hline Erythrocyte sedimentation rate (mm/hour) & $6(4$ to 13$)$ & 7 (4 to 22$)$ & $6(4$ to 11$)$ & 0.19 \\
\hline C-reactive protein (mg/l) & 5 (3 to 6$)$ & 5 (3 to 14$)$ & 5 (1 to 5$)$ & $<0.01$ \\
\hline Antinuclear antibody positivity & $79(36 \%)$ & $17(26 \%)$ & $62(41 \%)$ & 0.03 \\
\hline IgM-rheumatoid factor positivity & $11(5 \%)$ & $11(16 \%)$ & $0(0 \%)$ & $<0.01$ \\
\hline
\end{tabular}

Continuous variables are presented as median (interquartile range [IQR]). JRA, juvenile rheumatoid arthritis.

the medium genotype group and none in the low genotype group $(P=0.06)$. We did not find any association of MBL genotype groups with other clinical features, such as number of cumulative affected joints, arthritis severity index, PGA, $\mathrm{CHAQ}$ scores, or number of patients with uveitis, remission, or severe radiographic erosions, or with laboratory tests such as ANAs, erythrocyte sedimentation rate, and IgM-RF (Table 3). CRP levels were similar in the high, medium and low MBL2 genotype group (Table 3 ), even after stratification for remission status $(P>0.10$; Figure 2$)$. No differences in clinical or laboratory variables were found between patients with the $A$ / $A$, the $A / O$ and the $O / O M B L 2$ genotypes either (data not shown).

Oligoarthritis group

In the 151 oligoarthritis patients, age at onset was similar in the high, medium and low genotype expression groups $(P=$
0.66; Table 4). Patients with oligoarthritis carrying the low MBL expression genotype were more often in remission (81\%) than patients in the medium (54\%) and high (56\%) genotype groups $(P=0.02$; Table 4$)$. Multivariate analysis revealed that, after adjustment for disease duration, patients in the low genotype groups had an odds ratio of 2.5 (95\% confidence interval 1.1 to 5.7 ) of being in remission at follow up, as compared with patients in the high genotype group $(P=0.04$; data not shown). The median CRP level was $5 \mathrm{mg} / \mathrm{l}$ at follow up in the three genotype groups, but the CRP value distribution differed statistically significantly $(P<0.01$; Table 4$)$ between these three groups. Figure 2 shows CRP levels and MBL2 genotypes in patients with a current remission and patients with active disease with or without a previous remission. When the patients were stratified according to remission status (remission versus active), median CRP levels remained statistically significantly different in patients with active disease as com- 
Figure 1

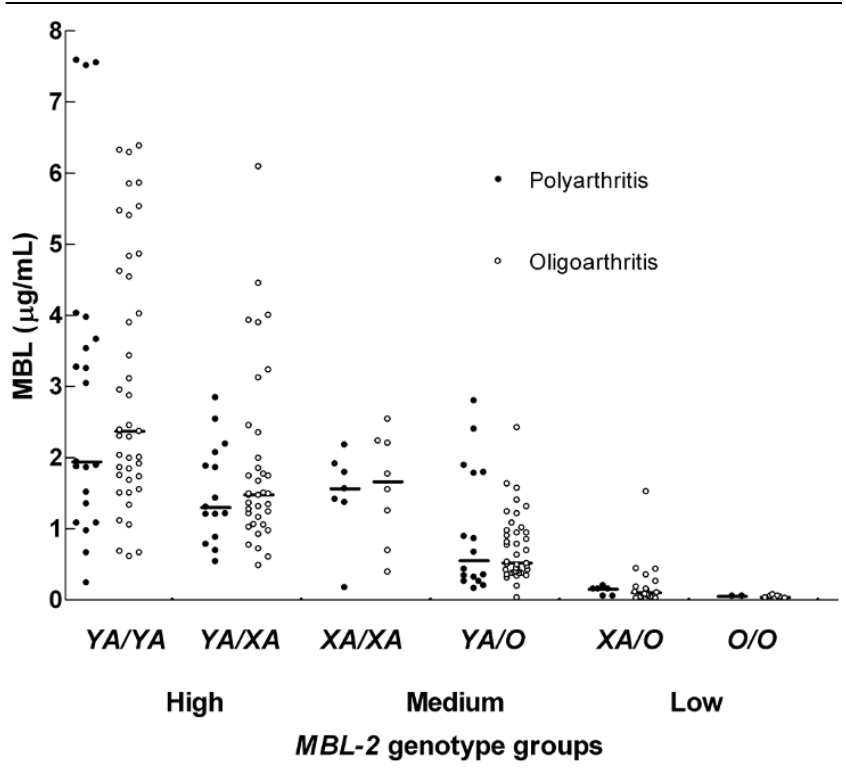

MBL level according to (extended) MBL2 haplotypes in patients with juvenile polyarthritis and oligoarthritis. Median mannose-binding lectin (MBL) plasma levels, represented by horizontal lines, differ between extended haplotype groups $(P<0.01)$, but not between patients with oligoarthritis $(n=151)$ and polyarthritis $(n=67)$ who had similar haplotypes $(P>0.46)$.

pared with those with a current remission. In these patients, the median (IQR) CRP level was 4 (1 to 5 ) $\mathrm{mg} / \mathrm{l}$ in the high genotype group versus 5 (4 to 10$) \mathrm{mg} / \mathrm{l}$ in the medium and 5 (5 to 9$) \mathrm{mg} / \mathrm{l}$ in the low genotype groups $(P<0.01)$.

The remaining clinical and laboratory variables did not differ between the patients in the high, medium and low MBL2 genotype groups (Table 4). The differences found in CRP level and remission status were also present in patients with the $A /$ $A$, the $A / O$ and the $O / O M B L 2$ genotype. Other clinical and laboratory variables did not differ between these patients (data not shown).

\section{Discussion}

In this study we demonstrated that the frequency of MBL deficiency was not increased in 218 Norwegian Caucasian children with JRA as compared with 194 Dutch Caucasian control individuals. Our observations are in agreement with the only previous study of MBL conducted in JIA patients [27]. In that study no association between MBL2 codon 54 mutations and JIA was found. We have now shown that JRA is also not associated with any of the other five known MBL2 SNPs.

The frequency of these mutations also did not differ from the frequencies identified in previously published Danish Caucasian control populations $[10,11]$. Over the past few years studies have been published that consistently reported similar frequencies in Caucasian populations of different countries $[10,11,34]$. Therefore, we assume that the frequencies of
MBL2 gene polymorphism in the Caucasian Norwegian population do not differ from those in other Caucasian populations. Therefore, our present observations suggest that genetically determined MBL deficiency is not associated with increased susceptibility to JRA. Based on the number of included patients and control individuals, this study has $80 \%$ power when an odds ratio of 1.71 or greater for MBL deficiency is found.

Interestingly, children in the low MBL2 genotype group developed polyarthritis at a younger age than did children in the medium or high genotype groups. Previously, Garred and coworkers [23] showed that MBL2 exon 1 variant allele carrier status was associated with early age at onset of RA, which is the adult counterpart of polyarthritis [26]. Garred and coworkers hypothesized that MBL may delay the onset of RA but that it does not prevent the disease. The mechanism by which MBL deficiency might promote inflammation in immune-mediated inflammatory diseases such as RA and JRA is as yet unknown. $\mathrm{MBL}$ deficiency might lead to a diminished innate immunity, and subsequent increased risk for infections, as was previously remonstrated $[15,16]$. These infections may trigger JRA, as has been hypothesized previously [26]. Another possibility is that MBL is involved in the recognition of an infectious agent in the pathophysiology of JRA. Low or absent MBL plasma concentration leads to decreased complement activation and ineffective clearance of the pathogen or pathogen-derived antigens. The prolonged presence of infectious agents in the host may enhance synovial inflammation because of the proinflammatory effects of bacterial DNA and bacterial cell wall fragments $[35,36]$. Anti-MBL autoantibodies may also play a role, because elevated levels of anti-MBL autoantibodies were found in the sera of RA patients [37]. It is unclear at present whether MBL deficiency is indeed involved in the pathogenesis of RA or JRA, because the data reported are variable.

Furthermore, MBL deficiency does not appear to play a role once polyarthritis has developed, because no associations were found between $M B L 2$ genotype and the laboratory variables or the remaining disease severity related clinical variables, such as PGA, CHAQ score, number of actively involved or affected joints, and number of patients with uveitis or remission. Consistent with the previous report by Barton and coworkers [25] on RA and MBL polymorphisms, we did not find an association between erosive joint destruction and MBL polymorphisms in patients with JRA.

In the oligoarthritis group, patients in the low genotype group were in remission more often (81\%) than were the children in the medium or high genotype group (54\% to $56 \%$ ). In this regard, lack of the protein MBL in serum appears to be associated with a milder disease course or decreased inflammation. The possible explanation for these findings might be that $M B L$ has an immunomodulating effect. MBL is present in synovial fluid and can bind potential causative agents in JRA 
Table 2

MBL concentrations and MBL2 genotypes

Control individuals

All JRA patients

81 (37)

$43(20)$

7 (3)

31 (14)

$A / D$

$20(10)$

9 (5)

4 (2)

$B / B$

$B / C$

$B / D$

$C / D$

$D / D$

Total

Genotype groups

$\begin{array}{ll}\text { High } & 110(57) \\ Y A / Y A & 60(31) \\ Y A / X A & 50(26) \\ \text { Medium } & 52(27) \\ X A / X A & 10(5) \\ Y A / O & 42(22) \\ \text { Low } & 32(16) \\ X A / O & 23(12) \\ \text { O/O } & 9(4)\end{array}$

Total

$194(100)$

MBL concentration

High

Medium

1.65 (1.20 to 2.69$)$

0.52 (0.40 to 0.92$)$

Low

$0.04(0.02$ to 0.13$)$
$9(4)$

4 (2)

$0(0)$

3 (1)

$1(0)$

$1(0)$

$218(100)$

$113(52)$

$62(28)$

51 (23)

71 (33)

$15(7)$

$56(26)$

25 (12)

9 (4)

$218(100)$

$1.86(1.23$ to 3.26$)$

0.77 (0.38 to 1.41$)$

0.07 (0.04 to 0.15$)$
34 (16)
JRA subgroups

Polyarthritis

Oligoarthritis

43(64)

85 (56)

22 (33)

59 (39)

$14(21)$

29 (19)

$0(0)$

7 (5)

8 (12)

23 (15)

2 (2)

7 (5)

1 (1)

3 (2)

$0(0)$

0 (0)

1 (1)

2 (1)

$0(0)$

1 (1)

$0(0)$

1 (1)

67 (100)

$151(100)$

36 (54)

77 (51)

21 (31)

41 (27)

15 (22)

36 (24)

23 (34)

48 (32)

7 (10)

8 (5)

$16(24)$

40 (27)

8 (12)

26 (17)

6 (9)

19 (13)

2 (3)

7 (5)

$67(100)$

$151(100)$

Norwegian Caucasian children with juvenile polyarthritis $(n=67)$ and oligoarthritis $(n=151)$ are compared with 194 healthy Dutch Caucasian adult control individuals. Values are expressed as number (\%) or, for continuous variables, as median (interquartile range). Median mannosebinding lectin (MBL) concentrations and frequencies of exon 1 mutations and MBL2 genotype groups did not differ between all juvenile

rheumatoid arthritis (JRA) patients and healthy control individuals or within the polyarthritis and oligoarthritis groups $(P$ values $>0.05)$. $A$ is the designation for wild-type; $O$ is the common designation for the variant alleles $B$ (codon 54), $C$ (codon 57 ) and $D$ (codon 52). 


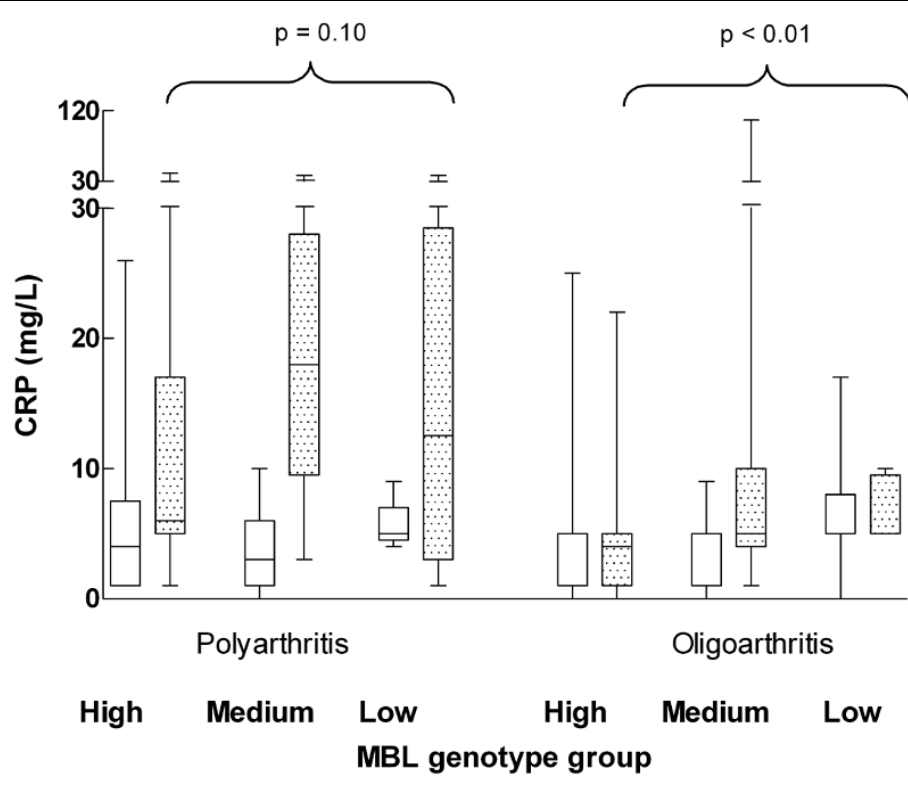

CRP and MBL2 genotype: remission versus active disease. Shown are serum C-reactive protein (CRP) concentrations (mg/l) and mannose-binding lectin (MBL) genotype in patients with a current remission versus active disease (either active disease with a previous remission or continuously active disease). *Only CRP values of oligoarthritis patients with active disease (as compared with patients with a current remission) differed statistically significantly $(P<0.01)$.

including micro-organisms, cellular debris, and agalactosyl $\lg G$ (IgG-G0) [38,39]. Binding of $M B L$ to agalactosyl IgG immune complexes may result in local complement activation and subsequent increased inflammation and thus active disease, whereas this is absent in the presence of very low levels of MBL [40]. Recently, Troelsen and colleagues [41] found that high serum levels of $\mathrm{MBL}$ and agalactosyl lgG were risk factors for ischaemic heart disease in RA patients. Besides, RA patients had higher MBL levels than did their relatives, suggesting that high MBL may trigger RA [39]. Harmful effects of high MBL levels have been shown in other disease entities as well. For instance, MBL deposits in the glomeruli can cause histological damage of kidneys, and activation of the lectin pathway by MBL can induce vascular tissue damage in myocardial ischemia-reperfusion injury and diabetes [42-44]. On the other hand, MBL deficiency might be associated with defective clearance of immune complexes and apoptotic cells, as seen in individuals with $\mathrm{C} 1 \mathrm{q}$ deficiency. Because MBL and $\mathrm{C} 1 \mathrm{q}$ are molecules with similar characteristics this might explain why during active disease CRP levels were increased in children in the low compared with the medium and high genotype groups. Remission rates were not associated with MBL2 genotype in patients with polyarthritis, possibly because more joints were affected.

\section{Conclusion}

MBL appears to play a dual role in JRA. Genetically determined MBL deficiency does not increase susceptibility to JRA, but MBL does appear to have an immunomodulating effect. On the one hand children with low levels of MBL develop pol- yarthritis at younger age. In the case of MBL deficiency, potential explanations for this younger age at onset are increased susceptibility to infections, as a potential trigger of polyarthritis, or ineffective clearance of infectious agents in the pathophysiology of JRA. On the other hand, the low MBL2 expressing genotypes appear to be beneficial once oligoarthritis has developed, because they are associated with increased frequency of remission. An explanation may be that the local MBL itself may lead to complement-mediated inflammation in the synovium, sustaining active disease. If we are to discover the possible contribution of MBL to JRA disease severity, then we must study molecular mechanisms such as the interaction of $\mathrm{MBL}$ with immune complexes, the presence of anti-MBL autoantibodies and the role of activation of the complement system.

\section{Competing interests}

The authors declare that they have no competing interests.

\section{Authors' contributions}

The study was designed by KD, TK, PT and AS. They were all involved in the management of the study and in supporting other contributors. BF, OF and AS collected the clinical data. NB conducted the laboratory investigations. FF analyzed the data statistically and interpreted the results. She completed the first draft, written by KD. Finally, each author contributed to the writing of the final manuscript. They all read and approved this version of the manuscript and take full responsibility for it. 
Table 3

Association of demographic, clinical, and laboratory characteristics and MBL2 genotype expression groups: juvenile polyarthritis

Characteristic

Age (years) at onset

Disease duration (years) at follow up

Clinical variables

Cumulative affected joints

Actively involved joints

Affected joints

Arthritis severity index

Physician global assessment

Childhood Health Assessment Questionnaire score

Patients with uveitis

Remission status at follow up

\section{Current remission}

Active, but previous remission

Continuously active

Radiographic erosions grade III to IV

Laboratory variables

Erythrocyte sedimentation rate $(\mathrm{mm} / \mathrm{hour})$

C-reactive protein $(\mathrm{mg} / \mathrm{l})$

Antinuclear antibody positivity

IgM-rheumatoid factor positivity
$M B L$ genotype expression groups

High $(n=36)$

$10(28 \%)$

9.5 (5.6 to 13.0 )

14.6 (13.5 to 16.3 )

8 (10 to 32$)$

1 (0 to 4 )

6 (2 to 18$)$

10 (2 to 31$)$

2 (1 to 3 )

0.1 (0.0 to 0.6$)$

$5(14 \%)$

$18(50 \%)$

$8(22 \%)$

$10(28 \%)$

$16(44 \%)$

8 (4 to 20 )

5 (3 to 9 )

$10(28 \%)$

$4(11 \%)$
$4(17 \%)$

10.1 (8.4 to 13.0 )

14.5 (13.2 to 16.4$)$

4.4 (3.6 to 7.0 )

2 (10 to 36$)$

2 (0 to 8 )

8 (0 to 20$)$

17 (0 to 46$)$

2 (1 to 4 )

0.3 (0.0 to 1.2$)$

$4(17 \%)$

$1(13 \%)$

NS NS

NS NS

NS NS

NS NS

NS NS

NS NS

NS NS

NS NS

NS NS
$4(50 \%)$

$2(25 \%)$

$4(17 \%)$

$2(25 \%)$

9 (39\%)

$4(50 \%)$

$10(44 \%)$

NS NS

NS NS

8 (5 to 25$)$

7 (3 to 18 )

3 (0 to 23$)$

5 (4 to 17$)$

$1(13 \%)$

$0(0 \%)$
$6(27 \%)$

7 (30\%)

.

Included in this analysis are 67 patients with juvenile polyarthritis. Values are expressed as number (\%) or, for continuous variables, as median (interquartile range). aComparison of the high, medium and low genotype expression groups by means of the two-sided Fisher's exact test and Kruskal-Wallis test. ${ }^{b}$ Comparison of the high and medium genotype group versus the low genotype expression group by means of the two-sided Fisher's exact test and Mann-Whitney U-test. NS, not significant. 


\section{Acknowledgements \\ Table 4}

\section{Association of demographic, clinical, and laboratory characteristics and MBL2 genotype expression groups: oligoarthritis}

Characteristic

Age (years) at onset

Disease duration (years) at follow up

Clinical variables

Cumulative affected joints

Actively involved joints

Affected joints

Arthritis severity index

Physician global assessment

Childhood Health Assessment Questionnaire score

Patients with uveitis

Remission status at follow up

Current remission

Active, but previous remission

Continuously active

Radiographic erosions grade III to IV

Laboratory variables

Erythrocyte sedimentation rate ( $\mathrm{mm} /$ hour)

C-reactive protein (mg/l)

Antinuclear antibody positivity

IgM-rheumatoid factor positivity
$M B L$ genotype expression groups

High $(n=77)$

$22(29 \%)$

6.6 (3.4 to 10.6$)$

$14.5(13.6$ to 15.9$)$

8.7 (2.6 to 12.1$)$

$8(31 \%)$

NS

NS

15.1 (13.2 to 16.1$) \quad 15.3$ (14.1 to 16.4$) \quad$ NS NS

$3(2$ to 6$)$

$0(0$ to 1$)$

1 (0 to 3$)$

$2(0$ to 6$)$

1 (1 to 2$)$

$0.0(0.0$ to 0.3$)$

$12(16 \%)$
$26(54 \%)$

$13(27 \%)$

$9(19 \%)$

$8(17 \%)$

$11(14 \%)$

6 (4 to 11$)$

8 (5 to 13$)$

5 (3 to 6)

$18(38 \%)$

$33(43 \%)$

0
NS NS

$0.02 \quad 0.01$

NS NS

NS NS

NS NS

NS NS

NS NS

NS NS

NS NS

$21(81 \%)$

$1(4 \%)$

$4(15 \%)$

$2(8 \%)$

NS NS

NS NS

NS NS

$5(5$ to 9$) \quad<0.01 \quad 0.01$

$11(42 \%) \quad$ NS NS

ncluded in this analysis are 151 patients with juvenile oligoarthritis. Values are expressed as number (\%) or, for continuous variables, as median (interquartile range). aComparison of the high, medium and low genotype expression groups by means of the two-sided Fisher's exact test and Kruskal-Wallis test. ${ }^{b}$ Comparison of the high and medium genotype group versus the low genotype expression group by means of the two-sided Fisher's exact test and Mann-Whitney U-test. NS, not significant. 
We thank Professor Ben Dijkmans for his intermediary support and Michel van Houdt for excellent technical assistance.

\section{References}

1. Burgos-Vargas R, Vazquez-Mellado J: The early clinical recognition of juvenile-onset ankylosing spondylitis and its differentiation from juvenile rheumatoid arthritis. Arthritis Rheum 1995, 38:835-844.

2. Ploski R, Vinje O, Ronningen KS, Spurkland A, Sorskaar D, Vartdal F, Forre O: HLA class II alleles and heterogeneity of juvenile rheumatoid arthritis. DRB1 ${ }^{\star} 0101$ may define a novel subset of the disease. Arthritis Rheum 1993, 36:465-472.

3. Schaller JG: Pauciarticular arthritis of childhood (pauciarticular juvenile rheumatoid arthritis). Ann Pediatr (Paris) 1983, 30:557-563.

4. Wilder RL, Crofford LJ: Do infectious agents cause rheumatoid arthritis? Clin Orthop Relat Res 1991, 265:36-41.

5. Turner MW: Mannose-binding lectin: the pluripotent molecule of the innate immune system. Immunol Today 1996, 17:532-540.

6. Neth O, Jack DL, Dodds AW, Holzel H, Klein NJ, Turner MW: Mannose-binding lectin binds to a range of clinically relevant microorganisms and promotes complement deposition. Infect Immun 2000, 68:688-693.

7. Saifuddin M, Hart ML, Gewurz H, Zhang Y, Spear GT: Interaction of mannose-binding lectin with primary isolates of human immunodeficiency virus type 1. J Gen Virol 2000, 81:949-955.

8. Lipscombe RJ, Sumiya M, Hill AV, Lau YL, Levinsky RJ, Summerfield JA, Turner MW: High frequencies in African and non-African populations of independent mutations in the mannose binding protein gene. Hum Mol Genet 1992, 1:709-715.

9. Madsen HO, Garred P, Kurtzhals JA, Lamm LU, Ryder LP, Thiel S, Svejgaard A: A new frequent allele is the missing link in the structural polymorphism of the human mannan-binding protein. Immunogenetics 1994, 40:37-44.

10. Brouwer N, Dolman KM, van Zwieten R, Nieuwenhuys E, Hart M, Aarden LA, Roos D, Kuijpers TW: Mannan-binding lectin (MBL)mediated opsonization is enhanced by the alternative pathway amplification loop. Mol Immunol 2006, 43:2051-2060.

11. Kronborg G, Weis N, Madsen HO, Pedersen SS, Wejse C, Nielsen $H$, Skinhoj $P$, Garred P: Variant mannose-binding lectin alleles are not associated with susceptibility to or outcome of invasive pneumococcal infection in randomly included patients. J Infect Dis 2002, 185:1517-1520.

12. Madsen HO, Garred $P$, Thiel S, Kurtzhals JA, Lamm LU, Ryder LP, Svejgaard $A$ : Interplay between promoter and structural gene variants control basal serum level of mannan-binding protein. $\mathrm{J}$ Immunol 1995, 155:3013-3020.

13. Madsen HO, Satz ML, Hogh B, Svejgaard A, Garred P: Different molecular events result in low protein levels of mannan-binding lectin in populations from southeast Africa and South America. J Immunol 1998, 161:3169-3175.

14. Frakking FN, van de Wetering $M D$, Brouwer $N$, Dolman KM, Geissler J, Lemkes B, Caron HN, Kuijpers TW: The role of mannose-binding lectin (MBL) in paediatric oncology patients with febrile neutropenia. Eur J Cancer 2006, 42:909-916.

15. Koch A, Melbye M, Sorensen P, Homoe P, Madsen HO, Molbak K, Hansen $\mathrm{CH}$, Andersen LH, Hahn GW, Garred P: Acute respiratory tract infections and mannose-binding lectin insufficiency during early childhood. JAMA 2001, 285:1316-1321.

16. Summerfield JA, Sumiya M, Levin M, Turner MW: Association of mutations in mannose binding protein gene with childhood infection in consecutive hospital series. BMJ 1997, 314:1229-1232.

17. Garred $\mathrm{P}$, Larsen F, Madsen HO, Koch C: Mannose-binding lectin deficiency - revisited. Mol Immunol 2003, 40:73-84

18. Lee YH, Witte T, Momot T, Schmidt RE, Kaufman KM, Harley JB, Sestak AL: The mannose-binding lectin gene polymorphisms and systemic lupus erythematosus: two case-control studies and a meta-analysis. Arthritis Rheum 2005, 52:3966-3974.

19. Graudal NA, Homann C, Madsen HO, Svejgaard A, Jurik AG, Graudal HK, Garred P: Mannan binding lectin in rheumatoid arthritis. A longitudinal study. J Rheumatol 1998, 25:629-635.

20. Graudal NA, Madsen HO, Tarp U, Svejgaard A, Jurik G, Graudal HK, Garred P: The association of variant mannose-binding lec- tin genotypes with radiographic outcome in rheumatoid arthritis. Arthritis Rheum 2000, 43:515-521.

21. Jacobsen $S$, Madsen HO, Klarlund M, Jensen T, Skjodt $H$, Jensen $\mathrm{KE}$, Svejgaard $A$, Garred $P$ : The influence of mannose binding lectin polymorphisms on disease outcome in early polyarthritis. TIRA Group. J Rheumato/ 2001, 28:935-942.

22. Saevarsdottir S, Vikingsdottir T, Vikingsson A, Manfredsdottir V Geirsson AJ, Valdimarsson $\mathrm{H}$ : Low mannose binding lectin predicts poor prognosis in patients with early rheumatoid arthritis. A prospective study. J Rheumatol 2001, 28:728-734.

23. Garred $\mathrm{P}$, Madsen HO, Marquart $\mathrm{H}$, Hansen TM, Sørensen SF, Petersen J, Volck B, Svejgaard A, Graudal NA, Rudd PM, Dwek RA, Sim RB, Andersen V: Two edged role of mannose binding lectin in rheumatoid arthritis: a cross sectional study. J Rheumatol 2000, 27:26-34.

24. Stanworth SJ, Donn RP, Hassall A, Dawes P, Ollier W, Snowden $\mathrm{N}$ : Absence of an association between mannose-binding lectin polymorphism and rheumatoid arthritis. $\mathrm{Br} J$ Rheumatol 1998 , 37:186-188.

25. Barton A, Platt $H$, Salway $F$, Symmons $D$, Lunt $M$, Worthington J, Silman A: Polymorphisms in the mannose binding lectin (MBL) gene are not associated with radiographic erosions in rheumatoid or inflammatory polyarthritis. J Rheumatol 2004, 31:442-447.

26. Ravelli A, Martini A: Juvenile idiopathic arthritis. Lancet 2007 , 369:767-778.

27. Kang M, Wang HW, Cheng PX, Yin ZD, Li XO, Shi H, Hu XF: Lack of association between mannose-binding lectin gene polymorphisms and juvenile idiopathic arthritis in a Han population from the Hubei province of China. Arthritis Res Ther 2006, 8:R85.

28. Flato B, Smerdel A, Johnston V, Lien G, Dale K, Vinje O, Egeland $\mathrm{T}$, Sorskaar D, Forre O: The influence of patient characteristics, disease variables, and HLA alleles on the development of radiographically evident sacroiliitis in juvenile idiopathic arthritis. Arthritis Rheum 2002, 46:986-994.

29. Flato B, Lien G, Smerdel A, Vinje O, Dale K, Johnston V, Sorskaar $\mathrm{D}$, Moum T, Ploski R, Forre O: Prognostic factors in juvenile rheumatoid arthritis: a case-control study revealing early predictors and outcome after 14.9 years. J Rheumatol 2003, 30:386-393

30. Brewer EJ Jr, Bass J, Baum J, Cassidy JT, Fink C, Jacobs J, Hanson V, Levinson JE, Schaller J, Stillman JS: Current proposed revision of JRA Criteria. JRA Criteria Subcommittee of the Diagnostic and Therapeutic Criteria Committee of the American Rheumatism Section of The Arthritis Foundation. Arthritis Rheum 1977 , 20:195-199.

31. Singh G, Athreya BH, Fries JF, Goldsmith DP: Measurement of health status in children with juvenile rheumatoid arthritis. Arthritis Rheum 1994, 37:1761-1769.

32. Tacx AN, Groeneveld AB, Hart MH, Aarden LA, Hack CE: Mannan binding lectin in febrile adults: no correlation with microbial infection and complement activation. J Clin Pathol 2003, 56:956-959.

33. Bernig T, Breunis W, Brouwer N, Hutchinson A, Welch R, Roos D, Kuijpers T, Chanock $S$ : An analysis of genetic variation across the MBL2 locus in Dutch Caucasians indicates that 3 ' haplotypes could modify circulating levels of mannose-binding lectin. Hum Genet 2005:1-12.

34. Garcia-Laorden MI, Pena MJ, Caminero JA, Garcia-Saavedra A Campos-Herrero Ml, Caballero A, Rodriguez-Gallego C: Influence of mannose-binding lectin on HIV infection and tuberculosis in a Western-European population. Mol Immunol 2006, 43:2143-2150.

35. Schrijver IA, Melief MJ, Tak PP, Hazenberg MP, Laman JD: Antigen-presenting cells containing bacterial peptidoglycan in synovial tissues of rheumatoid arthritis patients coexpress costimulatory molecules and cytokines. Arthritis Rheum 2000, 43:2160-2168.

36. van der Heijden I, Wilbrink B, Tchetverikov I, Schrijver IA, Schouls LM, Hazenberg MP, Breedveld FC, Tak PP: Presence of bacterial DNA and bacterial peptidoglycans in joints of patients with rheumatoid arthritis and other arthritides. Arthritis Rheum 2000, 43:593-598.

37. Gupta B, Raghav SK, Agrawal C, Chaturvedi VP, Das RH, Das HR: Anti-MBL autoantibodies in patients with rheumatoid arthritis: 
prevalence and clinical significance. J Autoimmun 2006, 27:125-133.

38. Saevarsdottir S, Vikingsdottir T, Valdimarsson $\mathrm{H}$ : The potential role of mannan-binding lectin in the clearance of self-components including immune complexes. Scand J Immunol 2004, 60:23-29

39. Saevarsdottir S, Steinsson K, Grondal G, Valdimarsson $H$ : Patients with rheumatoid arthritis have higher levels of mannan-binding lectin than their first-degree relatives and unrelated controls. J Rheumato/ 2007, 34:1692-1695.

40. Malhotra R, Wormald MR, Rudd PM, Fischer PB, Dwek RA, Sim RB: Glycosylation changes of IgG associated with rheumatoid arthritis can activate complement via the mannose-binding protein. Nat Med 1995, 1:237-243.

41. Troelsen LN, Garred P, Madsen HO, Jacobsen S: Genetically determined high serum levels of mannose-binding lectin and agalactosyl lgG are associated with ischemic heart disease in rheumatoid arthritis. Arthritis Rheum 2007, 56:21-29.

42. Roos A, Bouwman LH, Munoz J, Zuiverloon T, Faber-Krol MC, Fallaux-van den Houten FC, Klar-Mohamad N, Hack CE, Tilanus MG, Daha MR: Functional characterization of the lectin pathway of complement in human serum. Mol Immuno/ 2003, 39:655-668.

43. Hansen TK, Tarnow L, Thiel S, Steffensen R, Stehouwer CD, Schalkwijk CG, Parving HH, Flyvbjerg A: Association between mannose-binding lectin and vascular complications in type 1 diabetes. Diabetes 2004, 53:1570-1576.

44. Jordan JE, Montalto MC, Stahl GL: Inhibition of mannose-binding lectin reduces postischemic myocardial reperfusion injury. Circulation 2001, 104:1413-1418. 